\title{
Thermodynamics of Charged Rotating Dilaton Black Branes Coupled to Logarithmic Nonlinear Electrodynamics
}

\author{
A. Sheykhi, ${ }^{1,2}$ M. H. Dehghani, ${ }^{1,2}$ and M. Kord Zangeneh ${ }^{1}$ \\ ${ }^{1}$ Physics Department and Biruni Observatory, College of Sciences, Shiraz University, P.O. Box 55134-441, Shiraz 71454, Iran \\ ${ }^{2}$ Research Institute for Astronomy and Astrophysics of Maragha (RIAAM), P.O. Box 55134-441, Maragha, Iran \\ Correspondence should be addressed to A. Sheykhi; asheykhi@shirazu.ac.ir
}

Received 1 March 2016; Accepted 20 April 2016

Academic Editor: Elias C. Vagenas

Copyright (c) 2016 A. Sheykhi et al. This is an open access article distributed under the Creative Commons Attribution License, which permits unrestricted use, distribution, and reproduction in any medium, provided the original work is properly cited. The publication of this article was funded by $\mathrm{SCOAP}^{3}$.

\begin{abstract}
We construct a new class of charged rotating black brane solutions in the presence of logarithmic nonlinear electrodynamics with complete set of the rotation parameters in arbitrary dimensions. The topology of the horizon of these rotating black branes is flat, while due to the presence of the dilaton field the asymptotic behavior of them is neither flat nor (anti-)de Sitter [(A)dS]. We investigate the physical properties of the solutions. The mass and angular momentum of the spacetime are obtained by using the counterterm method inspired by AdS/CFT correspondence. We derive temperature, electric potential, and entropy associated with the horizon and check the validity of the first law of thermodynamics on the black brane horizon. We study thermal stability of the solutions in both canonical and grand-canonical ensemble and disclose the effects of the rotation parameter, nonlinearity of electrodynamics, and dilaton field on the thermal stability conditions. We find the solutions are thermally stable for $\alpha<1$, while for $\alpha>1$ the solutions may encounter an unstable phase, where $\alpha$ is dilaton-electromagnetic coupling constant.
\end{abstract}

\section{Introduction}

Thermodynamics of black holes plays a central role in the attractive modern method relating classical gravity and high energy physics, namely, gauge/gravity duality. The issue was first taken into consideration by Bekenstein [1] and Hawking [2] and encountered increasing interests rapidly. Among different frameworks, the thermodynamics of black solutions has been studied; dilaton gravity possesses a significant predominancy. This preference has at least two reasons. From one side, the dilaton gravity which is one of the modified gravities is able to justify the accelerating expansion of the universe confirmed from observations [3-9] while Einstein gravity (General Relativity) requires exotic matter violating energy conditions to justify this phase of universe. From another side, the dilaton gravity appears in the low energy limit of string theory [10] and therefore can provide a good laboratory for testing this theory in the low energy limit through gauge/gravity duality. Since string theory proposes higher than four dimensions [10], it is natural to consider higher dimensional solutions within the gravity theories come from string theory.

Exact asymptotically flat solutions of Einstein-Maxwelldilaton gravity have been constructed in the absence of dilaton potential in [11-20]. However, breaking of spacetime supersymmetry in ten dimensions may cause one or more Liouville-type potentials in the action of dilaton gravity. This type of dilaton potential changes the asymptotic behavior of solutions [17-19, 21-26]. In general, these solutions are neither asymptotically flat nor (anti-)de Sitter [(A)dS]. Thermodynamics of topological dilaton black holes in EinsteinMaxwell gravity has been explored in [27]. Asymptotically nonflat and non-(A)dS linearly charged rotating black branes were under investigation in $[28,29]$. Slowly rotating charged black holes have been studied from thermodynamics point of view as well [30]. 
A natural interesting extension of such solutions is to change the electrodynamics Lagrangian from linear Maxwell to nonlinear ones. Some efforts have been done to construct exact solution in dilaton gravity with nonlinear electrodynamics. For example, thermodynamics of static black hole solutions in the presence of nonlinear power-law Maxwell (PLM) [31], Born-Infeld (BI) [32], and exponential [33, 34] electrodynamics has been investigated. As well, thermodynamic properties of rotating black brane solutions have been studied in the presence of PLM [35], BI [36], and exponential [37] nonlinear electrodynamics. Any one of the abovementioned nonlinear electrodynamics has its own importance and motivations. For instance, the PLM electrodynamics extends the conformal invariance property of Linear Maxwell Lagrangian in four dimensions to higher dimensional spacetimes, while BI [38] electrodynamics, which comes from open string theory [39-43], solves the problem of infinite self-energy of charged point-particle that appears in linear Maxwell case. The latter problem is also overcome by logarithmic nonlinear electrodynamics proposed for the first time in [44]. Although this type of nonlinear electrodynamics has no direct relation with superstring theory, it can be motivated from different sides. First, it can be regarded as a toy model showing that certain nonlinear field theories can produce particle-like solutions that can realize the limiting curvature hypothesis in cosmological theories [44]. Second, the behavior of logarithmic electrodynamics and BI electrodynamics Lagrangians are the same for large values of nonlinear parameter $\beta$. Third, from gauge/gravity duality point of view, the ratio of holographic viscosity to entropy density is not affected by nonlinear terms rising from nonlinear electrodynamics in contrast with gravitational corrections [45]. Fourth, the values of important parameters of a holographic superconductor system such as critical temperature and order parameter are firmly sensitive to choice of electrodynamics $[46,47]$. Other studies on the nonlinear electrodynamics have been carried out in [36, 39-41, 48-59].

The above pointed out motivations are convincingly enough to satisfy one to seek for the effects of logarithmic electrodynamics on the solutions. Till now, exact rotating solutions of logarithmic electrodynamics in the context of dilaton gravity have not been constructed. In this paper, we would like to construct the rotating dilaton black branes in the presence of logarithmic nonlinear electrodynamics and investigate their thermodynamics as well as their thermal stability.

The outline of this paper is as follows. In Section 2, we present the basic field equations. In Section 3, we construct the rotating dilaton black branes with a complete set of rotation parameters in all higher dimensions and investigate their properties. In Section 4, we study thermodynamics of the spacetime, by calculating the conserved and thermodynamic quantities. In Section 5, we perform a stability analysis and show that the dilaton creates an unstable phase for the solutions. Section 6 is devoted to conclusions and discussions.

\section{Basic Field Equations}

We consider an $n$-dimensional action in which gravity is coupled to a dilaton and a nonlinear electrodynamic field

$$
\begin{aligned}
I= & -\frac{1}{16 \pi} \int_{\mathscr{M}} d^{n} x \sqrt{-g}\left(\mathscr{R}-\frac{4}{n-2}(\nabla \Phi)^{2}-V(\Phi)\right. \\
& +L(F, \Phi))-\frac{1}{8 \pi} \int_{\partial \mathscr{M}} d^{n-1} x \sqrt{-h} \Theta(h),
\end{aligned}
$$

where the Lagrangian of the logarithmic nonlinear electrodynamics coupled to the dilaton field (LNd) is chosen in the following form:

$$
L(F, \Phi)=-8 \beta^{2} e^{4 \alpha \Phi /(n-2)} \ln \left(1+\frac{e^{-8 \alpha \Phi /(n-2)} F^{2}}{8 \beta^{2}}\right) .
$$

In action (1), $\mathscr{R}$ is the Ricci scalar curvature, $\Phi$ is the dilaton field, and $V(\Phi)$ is a potential for $\Phi$. The dilaton parameter $\alpha$ determines the strength of coupling of the scalar and LNd fields, $F^{2}=F^{\mu \nu} F_{\mu \nu}$, where $F_{\mu \nu}=\partial_{\mu} A_{\nu}-\partial_{\nu} A_{\mu}$ is the electromagnetic tensor field, $A_{\mu}$ is the vector potential, and $\beta$ is the nonlinear parameter with dimension of mass. The last term in (1) is the Gibbons-Hawking boundary term which is chosen such that the variational principle is well-defined. The manifold $\mathscr{M}$ has metric $g_{\mu \nu}$ and covariant derivative $\nabla_{\mu}$. $\Theta$ is the trace of the extrinsic curvature $\Theta^{a b}$ of any boundary(ies) $\partial \mathscr{M}$ of the manifold $\mathscr{M}$, with induced metric(s) $h_{a b}$. In this paper, we consider action (1) with a Liouville-type potential,

$$
V(\Phi)=2 \Lambda e^{4 \alpha \Phi /(n-2)},
$$

where $\Lambda$ is a constant which may be referred to as the cosmological constant, since in the absence of the dilaton field $(\Phi=0)$ action (1) reduces to the action of Einstein gravity in the presence of nonlinear electrodynamics with cosmological constant. For later convenience, we redefine it as $\Lambda=-(n-1)(n-2) / 2 l^{2}$, where $l$ is a constant with dimension of length. The series expansion of (2) for large $\beta$ leads to

$$
\begin{aligned}
L_{\mathrm{LNd}}(F, \Phi)= & -e^{-4 \alpha \Phi /(n-2)} F^{2}+\frac{e^{-12 \alpha \Phi /(n-2)} F^{4}}{16 \beta^{2}} \\
& -\frac{e^{-20 \alpha \Phi /(n-2)} F^{6}}{192 \beta^{4}}+\mathcal{O}\left(\frac{1}{\beta^{6}}\right) .
\end{aligned}
$$

For later convenience we rewrite

$$
L_{\mathrm{LNd}}(F, \Phi)=-8 \beta^{2} e^{4 \alpha \Phi /(n-2)} \mathscr{L}(Y),
$$

where we have defined

$$
\begin{aligned}
\mathscr{L}(Y) & =\ln (1+Y), \\
Y & =\frac{e^{-8 \alpha \Phi /(n-2)} F^{2}}{8 \beta^{2}} .
\end{aligned}
$$


By varying action (1) with respect to the gravitational field $g_{\mu \nu}$, the dilaton field $\Phi$, and the gauge field $A_{\mu}$, we find

$$
\begin{aligned}
& \mathscr{R}_{\mu \nu} \\
&= \frac{4}{n-2}\left(\partial_{\mu} \Phi \partial_{\nu} \Phi+\frac{1}{4} g_{\mu \nu} V(\Phi)\right) \\
&+2 e^{-4 \alpha \Phi /(n-2)} \partial_{Y} \mathscr{L}(Y) F_{\mu \eta} F_{\nu}{ }^{\eta} \\
& \quad-\frac{8 \beta^{2}}{n-2} e^{4 \alpha \Phi /(n-2)}\left[2 Y \partial_{Y} \mathscr{L}(Y)-\mathscr{L}(Y)\right] g_{\mu \nu}, \\
& \nabla^{2} \Phi \quad \frac{n-2}{8} \frac{\partial V}{\partial \Phi} \\
& \quad-4 \alpha \beta^{2} e^{4 \alpha \Phi /(n-2)}\left[2 Y \partial_{Y} \mathscr{L}(Y)-\mathscr{L}(Y)\right], \\
& \nabla_{\mu}\left(e^{-4 \alpha \Phi /(n-2)} \partial_{Y} \mathscr{L}(Y) F^{\mu \nu}\right)=0 .
\end{aligned}
$$

In the limiting case where $\beta \rightarrow \infty$, we have $\mathscr{L}(Y)=Y$. In this case the system of field equations (7)-(9) restore the wellknown equations of EMd gravity [21-27], as expected.

\subsection{Finite Action in Canonical and Grand-Canonical Ensem-} bles. In general, the total action I given in (1) is divergent when evaluated on a solution. One way of dealing with the divergences of the action is adding some counterterms to action (1). The counterterms should contain a part which removes the divergence of the gravity part of the action and a part for dealing with the divergence of the matter action. Since the horizon of our solution is flat, the counterterm which removes the divergence of the gravity part should be proportional to $\sqrt{-h}$. The counterterm for the matter part of the action in the presence of the dilaton is given by

$$
I_{\mathrm{ct}}=-\frac{1}{8 \pi} \int_{\partial \mathscr{M}} d^{n-1} x \sqrt{-h}\left(\frac{n-2}{l_{\mathrm{eff}}}\right)+I_{\mathrm{deriv}},
$$

where $l_{\text {eff }}$ is given by (16) and $I_{\text {deriv }}$ is a collection of terms involving derivatives of the boundary fields that could involve the curvature tensor constructed from the boundary metric. Since in our case the boundary is flat so $I_{\text {deriv }}$ is zero on the boundary. The variation of the total action $\left(I_{\text {tot }}=I+I_{\mathrm{ct}}\right)$ about the solutions of the equations of motion is

$$
\begin{aligned}
& \delta I_{\text {tot }} \\
& =\int d^{n-1} x S_{a b} \delta h^{a b} \\
& \quad-\frac{1}{16 \pi} \int d^{n-1} x \sqrt{-h} e^{-4 \alpha \Phi /(n-2)} \partial_{Y} \mathscr{L}(Y) n^{a} F_{a b} \delta A^{b},
\end{aligned}
$$

where

$$
S_{a b}=\frac{\sqrt{-h}}{16 \pi}\left\{\Theta_{a b}-\Theta h_{a b}+\frac{n-2}{l_{\mathrm{eff}}} h_{a b}\right\} .
$$

Equation (11) shows that the variation of the total action with respect to $A^{a}$ will only give the equation of motion of the nonlinear massless field $A^{a}$ provided the variation is at fixed nonlinear massless gauge potential on the boundary. Thus, the total action, $I_{\text {tot }}=I+I_{\mathrm{ct}}$, given in (11) is appropriate for the grand-canonical ensemble, where $\delta A^{a}=0$ on the boundary. But in the canonical ensemble, where the electric charge $\left[-e^{-4 \alpha \Phi /(n-2)} \partial_{Y} \mathscr{L}(Y) n^{a} F_{a b}\right]$ is fixed on the boundary, the appropriate action is

$$
\begin{aligned}
& I_{\mathrm{tot}}=I+I_{\mathrm{ct}}+\frac{1}{16 \pi} \\
& \cdot \int_{\partial \mathscr{M}^{c}} d^{n-1} x \sqrt{-h} e^{-4 \alpha \Phi /(n-2)} \partial_{Y} \mathscr{L}(Y) n^{a} F_{a b} \delta A^{b} .
\end{aligned}
$$

The last term in (13) is the generalization of the boundary term introduced by Hawking and Ross for linear electromagnetic field $[60]$ and the results of $[61,62]$ for the nonlinear Lifshitz black holes to the exponential nonlinear gauge field coupled to the dilaton field. Thus, both in canonical and grand-canonical ensemble, the variation of total action about the solutions of the field equations is

$$
\delta I_{\text {tot }}=\int d^{n-1} x S_{a b} \delta h^{a b} .
$$

That is, the nonlinear gauge field is absent in the variation of the total action both in canonical and grand-canonical ensembles.

In order to obtain the conserved charges of the spacetime, we use the counterterm method [63-67] inspired by (A)dS/CFT correspondence. For asymptotically AdS solutions this method works very well [64-67]. However, in our paper we have the scalar dilaton field with a Liouville potential. It was argued that the presence of Liouville-type dilaton potential, which is regarded as the generalization of the cosmological constant, changes the asymptotic behavior of the solutions to be neither asymptotically flat nor (A)dS. It has been shown that no dilaton dS or AdS black hole solution exists with the presence of only one Liouville-type dilaton potential [17-19]. But, as in the case of asymptotically AdS spacetimes, according to the domain-wall/QFT (quantum field theory) correspondence [68-70], there may be a suitable counterterm for the stress-energy tensor which removes the divergences. In this paper, we deal with the spacetimes with zero curvature boundary $\left[R_{a b c d}(h)=0\right]$, and therefore the counterterm for the stress-energy tensor should be proportional to $h^{a b}$. We find the finite stress-energy tensor in $n$ dimensional Einstein-dilaton gravity with Liouville-type in the form $[36,59]$

$$
T^{a b}=\frac{1}{8 \pi}\left[\Theta^{a b}-\Theta h^{a b}+\frac{n-2}{l_{\mathrm{eff}}} h^{a b}\right],
$$

where $l_{\text {eff }}$ is given by

$$
l_{\text {eff }}^{2}=\frac{(n-2)\left(\alpha^{2}-n+1\right)}{2 \Lambda} e^{-4 \alpha \Phi /(n-2)} .
$$

In the particular case $\alpha=0$, the effective $l_{\text {eff }}^{2}$ of (16) reduces to $l^{2}=-(n-1)(n-2) / 2 \Lambda$ of the AdS spacetimes. 
The first two terms in (15) are the variation of action (1) with respect to $h_{a b}$, and the last term is the counterterm which removes the divergences. One may note that the counterterm has the same form as in the case of asymptotically AdS solutions with zero curvature boundary, where $l$ is replaced by $l_{\text {eff }}$. If we choose the Killing vector field $\xi$ on spacelike surface $\mathscr{B}$ in $\partial \mathscr{M}$ with metric $\sigma_{i j}$, then the quasilocal conserved quantities may be obtained from the following relation $[36,59]$ :

$$
Q(\xi)=\int_{\mathscr{B}} d^{n-2} x \sqrt{\sigma} T_{a b} n^{a} \xi^{b}
$$

where $\sigma$ is the determinant of the boundary metric $\sigma_{i j}$ and $n^{a}$ is the unit normal vector on the boundary $\mathscr{B}$. In our case, the boundary $\mathscr{B}$ has two Killing vector fields timelike $(\partial / \partial t)$ and rotational $(\partial / \partial \varphi)$. The corresponding conserved charges are the quasilocal mass and angular momentum may be obtained as

$$
\begin{aligned}
M & =\int_{\mathscr{B}} d^{n-2} x \sqrt{\sigma} T_{a b} n^{a} \xi^{b}, \\
J & =\int_{\mathscr{B}} d^{n-2} x \sqrt{\sigma} T_{a b} n^{a} \varsigma^{b} .
\end{aligned}
$$

\section{Rotating Dilaton Black Branes in Higher Dimensions}

In this section, we would like to construct the rotating black brane solutions of the field equations (7)-(9) with $k$ rotation parameters. The number of independent rotation parameters for an $n$-dimensional localized object is equal to the number of Casimir operators, which is $[(n-1) / 2] \equiv k$, where $[x]$ is the integer part of $x$ [71]. The metric of $n$-dimensional rotating solution with cylindrical or toroidal horizons and $k$ rotation parameters can be written as $[72,73]$

$$
\begin{aligned}
d s^{2}= & -f(r)\left(\Xi d t-\sum_{i=1}^{k} a_{i} d \phi_{i}\right)^{2} \\
& +\frac{r^{2}}{l^{4}} R^{2}(r) \sum_{i=1}^{k}\left(a_{i} d t-\Xi l^{2} d \phi_{i}\right)^{2} \\
& -\frac{r^{2}}{l^{2}} R^{2}(r) \sum_{i<j}^{k}\left(a_{i} d \phi_{j}-a_{j} d \phi_{i}\right)^{2}+\frac{d r^{2}}{f(r)} \\
& +\frac{r^{2}}{l^{2}} R^{2}(r) d X^{2}, \\
\Xi^{2}= & +\sum_{i=1}^{k} \frac{a_{i}^{2}}{l^{2}},
\end{aligned}
$$

where $a_{i}$ 's are $k$ rotation parameters. There are two unknown functions $f(r)$ and $R(r)$ in the above metric which should be determined by solving the field equations. The range of the angular coordinates is $0 \leq \phi_{i} \leq 2 \pi$ and $d X^{2}$ is the Euclidean metric on the $(n-k-2)$-dimensional submanifold with volume $\Sigma_{n-k-2}$.
First of all, we integrate the electromagnetic field equation (9). The result is

$$
\begin{aligned}
F_{t r} & =\frac{2 q \Xi e^{4 \alpha \Phi /(n-2)}}{(r R(r))^{n-2}}\left(1+\sqrt{1+\frac{q^{2}}{\beta^{2}(r R(r))^{2 n-4}}}\right)^{-1}, \\
F_{\phi_{i} r} & =-\frac{a_{i}}{\Xi} F_{t r},
\end{aligned}
$$

where $q$ is an integration constant related to the electric charge of the brane. When $\beta \rightarrow \infty, F_{t r}$ reduces to the electric field of $n$-dimensional black brane of Einstein-Maxwelldilaton gravity [71]

$$
F_{t r}=\frac{q \Xi e^{4 \alpha \Phi /(n-2)}}{(r R(r))^{n-2}}+O\left(\frac{1}{\beta^{2}}\right) .
$$

In order to solve the system of (7) and (8) for three unknown functions $f(r), R(r)$, and $\Phi(r)$, we make the ansatz [71]

$$
R(r)=e^{2 \alpha \Phi /(n-2)} .
$$

In order to justify this choice for the metric function $R(r)$, let us note that $R(r)$ is indeed added to the metric (20) in order to increase the degrees of freedom for obtaining solutions in the presence of the dilaton field. Choosing $R(r)$ in the form of (23) is an ansatz. However, it is chosen such that, in the absence of the dilaton field $\Phi=0$, we have $R(r)=1$, as expected. With this ansatz, we are able to solve the field equation, analytically.

Substituting (23), the electromagnetic fields (21), and the metric (20) into the field equations (7) and (8), one can obtain the following solutions:

$$
\begin{aligned}
& f(r)=\frac{2\left(\alpha^{2}+1\right)^{2}\left(\Lambda-4 \beta^{2}\right) b^{\gamma}}{(n-2)\left(\alpha^{2}-n+1\right)} r^{2-\gamma}-\frac{m}{r^{n-3-(n-2) \gamma / 2}} \\
& -\frac{8 \beta^{2}\left(\alpha^{2}+1\right) b^{\gamma}}{(n-2) r^{n-3-(n-2) \gamma / 2}} \int r^{n(1-\gamma / 2)-2}\{\sqrt{1+\eta} \\
& \left.-\ln \left(\frac{\eta}{2}\right)+\ln (-1+\sqrt{1+\eta})\right\} d r, \\
& \Phi(r)=\frac{(n-2) \alpha}{2\left(\alpha^{2}+1\right)} \ln \left(c+\frac{b}{r}\right),
\end{aligned}
$$

where $c$ and $b$ are constant of integration. We find that these solutions will fully satisfy the system of (7) and (8) provided we choose $c=0$. Note that $b$ has the dimension of [Length] to make the argument of logarithmic function dimensionless. In the above solutions $\gamma=2 \alpha^{2} /\left(1+\alpha^{2}\right)$, and

$$
\eta=\frac{q^{2} b^{(2-n) \gamma}}{\beta^{2} r^{(n-2)(2-\gamma)}} .
$$

In the above expression, $m$ appears as an integration constant and is related to the mass of the black hole. The integration of 
(24) can be performed using the MATHEMATICA software. The resulting solution can be written

$$
\begin{aligned}
& f(r)=\frac{2\left(\Lambda-4 \beta^{2}\right)\left(\alpha^{2}+1\right)^{2} b^{\gamma}}{(n-2)\left(\alpha^{2}-n+1\right)} r^{2-\gamma}-\frac{m}{r^{n-3-(n-2) \gamma / 2}} \\
& +\frac{8 \beta^{2}\left(\alpha^{2}+1\right)^{2}}{\left(\alpha^{2}-n+1\right)^{2}} b^{\gamma} r^{2-\gamma}\{1 \\
& \left.-{ }_{2} F_{1}\left(\left[\frac{-1}{2}, \frac{\alpha^{2}-n+1}{2 n-4}\right],\left[\frac{\alpha^{2}+n-3}{2 n-4}\right],-\eta\right)\right\} \\
& +\frac{8 \beta^{2}\left(\alpha^{2}+1\right)^{2}}{(n-2)\left(\alpha^{2}-n+1\right)} b^{\gamma} r^{2-\gamma}\left\{\sqrt{1+\eta}-\ln \left(\frac{\eta}{2}\right)\right. \\
& +\ln (-1+\sqrt{1+\eta})\},
\end{aligned}
$$

where ${ }_{2} F_{1}([a, b],[c], z)$ is the hypergeometric function [74, 75]. It is worth mentioning that the solutions are ill-defined for $\alpha=\sqrt{n-1}$. We expect that, for large $\beta$, the function $f(r)$ reduces to the $n$-dimensional charged rotating dilaton black brane solutions given in [71]. Indeed, if we expand (27) for large $\beta$, we arrive at

$$
\begin{aligned}
f(r)= & \frac{2 \Lambda\left(\alpha^{2}+1\right)^{2}}{(n-2)\left(\alpha^{2}-n+1\right)} b^{\gamma} r^{2-\gamma}-\frac{m}{r^{n-3-(n-2) \gamma / 2}} \\
& +\frac{2 q^{2}\left(\alpha^{2}+1\right)^{2} b^{-(n-3) \gamma}}{(n-2)\left(\alpha^{2}+n-3\right) r^{(n-3)(2-\gamma)}} \\
& -\frac{q^{4}\left(\alpha^{2}+1\right)^{2} b^{-(2 n-5) \gamma}}{4 \beta^{2}(n-2)\left(\alpha^{2}+3 n-7\right) r^{(2 n-5)(2-\gamma)}} \\
& +\mathcal{O}\left(\frac{1}{\beta^{4}}\right) .
\end{aligned}
$$

Setting $\alpha=\gamma=0$ in (28), we reach

$$
\begin{aligned}
f(r)= & \frac{r^{2}}{l^{2}}-\frac{m}{r^{n-3}}+\frac{2 q^{2}}{(n-2)(n-3) r^{2 n-6}} \\
& -\frac{1}{4 \beta^{2}(n-2)(3 n-7)} \frac{q^{4}}{r^{4 n-10}}+\mathcal{O}\left(\frac{1}{\beta^{4}}\right) .
\end{aligned}
$$

The last term in the right hand side of the above expression is the leading nonlinear correction to the AdS black brane with dilaton field. In the absence of a nontrivial dilaton $(\alpha=$ $\gamma=0$ ), the above solutions reduce to the asymptotically AdS charged rotating black brane solutions of Einstein gravity in the presence of EN electrodynamics $[52,53]$. Finally, in the limit $\beta^{2} \rightarrow \infty$ and $\alpha=0=\gamma$, the solution given by (28) has the form of the asymptotically AdS black brane solutions $[72,76,77]$. Figures 1 and 2 depict the behavior of $f(r)$ given by (27) for different $\alpha$ 's and $\beta$ 's, respectively.

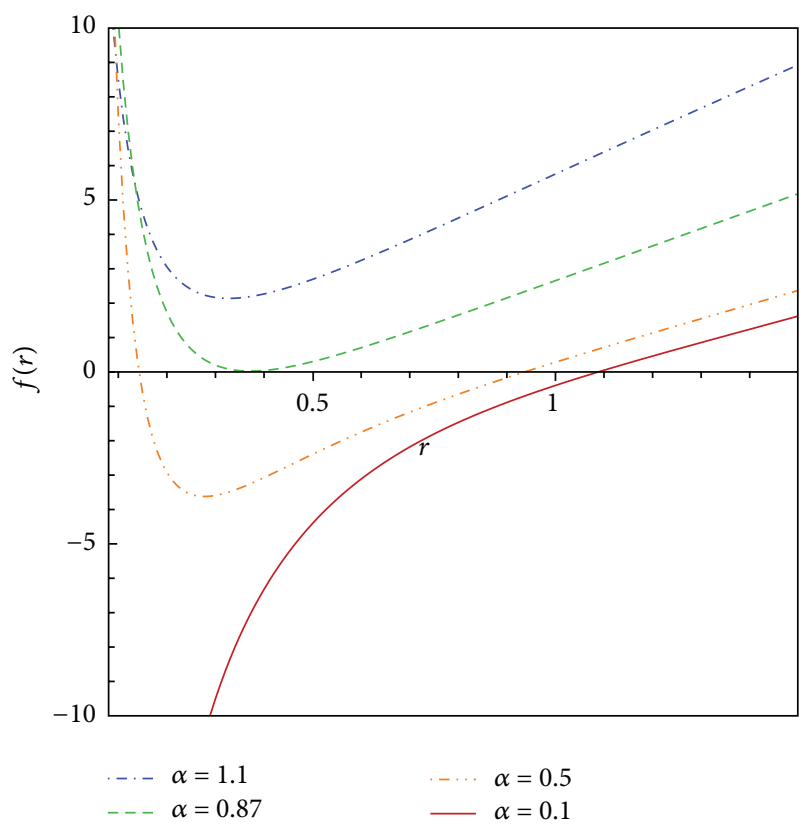

FIgURE 1: The behavior of $f(r)$ versus $r$ with $l=b=1, q=0.5$, $\Xi=1.25, n=5, \beta=2$, and $m=1.5$.

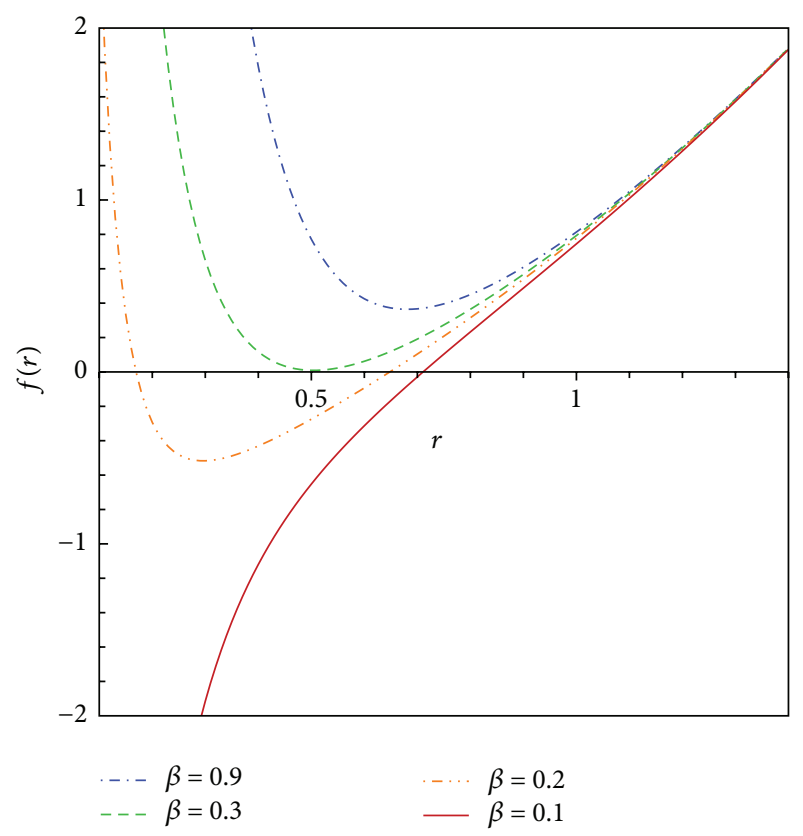

FIGURE 2: The behavior of $f(r)$ versus $r$ with $l=b=1, q=0.8$, $\Xi=1.25, n=5, \alpha=0.2$, and $m=0.5$.

3.1. Asymptotic Behavior of the Spacetime. Next, we study the geometry of this spacetime. For this purpose, we first seek for the curvature singularities in the presence of dilaton and nonlinear electrodynamic fields. It is a matter of calculation 
to show that the Ricci scalar and the Kretschmann invariant behave as

$$
\begin{aligned}
\lim _{r \rightarrow 0^{+}} R & =\infty, \\
\lim _{r \rightarrow 0^{+}} R_{\mu \nu \rho \sigma} R^{\mu \nu \rho \sigma} & =\infty,
\end{aligned}
$$

which indicate that there is an essential singularity at $r=0$. In order to study the asymptotic behavior of the solutions, we expand the metric function $f(r)$ for $r \rightarrow \infty$ limit. We find

$$
\lim _{r \rightarrow \infty} f(r)=\frac{2 \Lambda\left(\alpha^{2}+1\right)^{2}}{(n-2)\left(\alpha^{2}-n+1\right)} b^{\gamma} r^{2-\gamma} .
$$

Let us note that, in the absence of the dilaton field $(\alpha=0=\gamma)$, the metric function becomes

$$
\lim _{r \rightarrow \infty} f(r)=-\frac{2 \Lambda r^{2}}{(n-1)(n-2)},
$$

which describes asymptotically $\operatorname{AdS}(\Lambda<0)$ or dS $(\Lambda>0)$ spacetimes. However, as one can see from (31), in the presence of the dilaton field, the asymptotic behavior is neither flat nor (A)dS. For example, taking $\alpha=\sqrt{2}, n=6$, and $b=1$, we have

$$
\lim _{r \rightarrow \infty} f(r)=-\frac{3 \Lambda}{2} r^{2 / 3}
$$

Clearly, the metric function (33) is neither flat nor (A)dS. This is consistent with the argument given in [17-19], which states that no dilaton $\mathrm{dS}$ or AdS black hole solution exists with the presence of only one or two Liouville-type dilaton potentials. It is important to note that this asymptotic behavior is not due to the nonlinear nature of the electrodynamic field, since as $r \rightarrow \infty$ the effects of the nonlinearity disappear. Besides, from the dilaton field (25) we see that as $r \rightarrow \infty$, the dilaton field does not vanish, while in case of asymptotic flat or (A)dS we expect to have $\lim _{r \rightarrow \infty} \Phi(r)=0$. Indeed, by solving the field equation (8) we find

$$
\Phi(r)=\frac{(n-2) \alpha}{2\left(\alpha^{2}+1\right)} \ln \left(c+\frac{b}{r}\right)
$$

however, the system of (7)-(9) will be fully satisfied provided we choose $c=0$. From the above arguments we conclude that the asymptotic behavior of the obtained solutions is neither flat nor (A)dS.

\section{Thermodynamics of Black Branes}

It is easy to show that the metric given by (20) and (27) has both Killing and event horizons [71]. The Killing horizon is a null surface whose null generators are tangent to a Killing field. It is easy to see that the Killing vector,

$$
\chi=\partial_{t}+\sum_{i=1}^{k} \Omega_{i} \partial_{\phi_{i}}
$$

is the null generator of the event horizon, where $\Omega_{i}$ is the $i$ th component of angular velocity of the outer horizon which may be obtained by analytic continuation of the metric. The Hawking temperature and the angular velocities of the outer event horizon can be obtained as

$$
\begin{aligned}
T_{+} & =\frac{f^{\prime}\left(r_{+}\right)}{4 \pi \Xi}=-\frac{\alpha^{2}+1}{4 \pi \Xi} r_{+}^{1-\gamma}\left\{\frac{2\left(\Lambda-4 \beta^{2}\right) b^{\gamma}}{(n-2)}\right. \\
& +\frac{8 \beta^{2} b^{\gamma}}{n-2}\left[\sqrt{1+\eta_{+}}-\ln \left(\frac{\eta_{+}}{2}\right)\right. \\
& \left.\left.+\ln \left(-1+\sqrt{1+\eta_{+}}\right)\right]\right\}, \\
\Omega_{i} & =\frac{a_{i}}{\Xi l^{2}},
\end{aligned}
$$

where $\eta_{+}=\eta\left(r=r_{+}\right)$and we have used $f\left(r_{+}\right)=0$ for deleting $m$. For large $\beta$, we can expand $T_{+}$and arrive at the temperature of the higher dimensional black branes in EMd gravity [71]:

$$
\begin{aligned}
T_{+}= & -\frac{\Lambda\left(\alpha^{2}+1\right) b^{\gamma}}{2 \pi \Xi(n-2)} r_{+}^{1-\gamma} \\
& -\frac{q^{2}\left(\alpha^{2}+1\right) b^{-\gamma(n-3)}}{2 \pi \Xi(n-2)} r_{+}^{5-2 n-3 \gamma+n \gamma}+\mathcal{O}\left(\frac{1}{\beta^{2}}\right) .
\end{aligned}
$$

The mass and angular momentum of the black branes ( $\alpha<\sqrt{n-1}$ ) can be calculated through the use of (18). Denoting the volume of the hypersurface boundary at constant $t$ and $r$ by $V_{n-2}=(2 \pi)^{k} \Sigma_{n-k-2}$, the mass and angular momentum per unit volume $V_{n-2}$ of the black branes can be obtained as

$$
\begin{aligned}
M & =\frac{b^{(n-2) \gamma / 2}}{16 \pi l^{n-3}}\left\{\frac{\left(n-1-\alpha^{2}\right) \Xi^{2}+\alpha^{2}-1}{1+\alpha^{2}}\right\} m, \\
J_{i} & =\frac{b^{(n-2) \gamma / 2}}{16 \pi l^{n-3}}\left(\frac{n-1-\alpha^{2}}{1+\alpha^{2}}\right) \Xi m a_{i} .
\end{aligned}
$$

Note that, in order to avoid repeating the factor $V_{n-2}$, we calculate, in this paper, the mass $M$ and extensive quantities such as angular momentum $J_{i}$, entropy $S$, and charge $Q$ appearing in first law of thermodynamics per unit volume. For the static case where $a_{i}=0(\Xi=1)$, the angular momentum per unit volume vanishes, and therefore $a_{i}$ 's are the rotational parameters of the black branes.

Black hole entropy typically satisfies the so-called area law of the entropy $[2,78,79]$. This near universal law applies to almost all kinds of black holes and black branes in Einstein gravity [80-83]. It is easy to show that the entropy per unit volume $V_{n-2}$ of the black brane can be written as

$$
S=\frac{\Xi b^{(n-2) \gamma / 2} r_{+}^{(n-2)(1-\gamma / 2)}}{4 l^{n-3}} .
$$


The electric charge per unit volume $V_{n-1}$ can be found by calculating the flux of the electric field at infinity, yielding

$$
\begin{aligned}
Q & =-\frac{1}{4 \pi V_{n-1}} \int_{\Sigma} \nabla_{\mu}\left(\partial_{Y} \mathscr{L}(Y) F^{\mu \nu}\right) d S_{\nu} \\
& =-\frac{1}{8 \pi V_{n-1}} \oint_{\partial \Sigma} \partial_{Y} \mathscr{L}(Y) F^{\mu \nu} d S_{\mu \nu}=\frac{\Xi q}{4 \pi l^{n-3}},
\end{aligned}
$$

where the volume is replaced by an arbitrary spacelike hypersurface $\Sigma$ (partial Cauchy surface) with boundary $\partial \Sigma$. In addition, the volume element on $\Sigma$ is a nonspacelike covector (1-form) $d S_{\nu}$ and $d S_{\mu \nu}$ is the area element of $\partial \Sigma$. We should note that, for linear Maxwell case $(\beta \rightarrow \infty)$, one obtains $\partial_{Y} \mathscr{L}(Y)=-1$.

The electric potential $U$, measured at infinity with respect to the horizon, is defined by

$$
U=\left.A_{\mu} \chi^{\mu}\right|_{r \rightarrow \infty}-\left.A_{\mu} \chi^{\mu}\right|_{r=r_{+}}
$$

where $\chi$ is the null generator of the horizon given by (35). One can easily show that the vector potential $A_{\mu}$ corresponding to the electromagnetic tensor (21) can be written as

$$
\begin{aligned}
& A_{\mu}=\left(\Xi \delta_{\mu}^{t}-a_{i} \delta_{\mu}^{i}\right) \frac{q\left(\alpha^{2}+1\right) b^{(4-n) \gamma / 2}}{\alpha^{2}+n-3} \\
& \cdot r^{3-n-(4-n) \gamma / 2}{ }_{3} F_{2}\left(\left[\frac{1}{2}, 1, \frac{3-n-\alpha^{2}}{4-2 n}\right],\right. \\
& \\
& {\left.\left[2, \frac{7-3 n-\alpha^{2}}{4-2 n}\right],-\eta\right), }
\end{aligned}
$$

where ${ }_{3} F_{2}$ is the hypergeometric function and we have set the constant of integration equal to zero. Therefore, the electric potential may be obtained as

$$
\begin{aligned}
U & =\frac{q\left(\alpha^{2}+1\right) b^{(4-n) \gamma / 2}}{\Xi\left(\alpha^{2}+n-3\right)} \\
& \cdot r_{+}^{3-n-(4-n) \gamma / 2}{ }_{3} F_{2}\left(\left[\frac{1}{2}, 1, \frac{3-n-\alpha^{2}}{4-2 n}\right],\right. \\
& {\left.\left[2, \frac{7-3 n-\alpha^{2}}{4-2 n}\right],-\eta_{+}\right) . }
\end{aligned}
$$

Now, we are in a position to verify the first law of thermodynamics. In order to do this, we obtain the mass $M$ as a function of extensive quantities $S$, J, and $Q$. Using the expression for the mass, the angular momenta, the entropy, and the charge given in (38), (39), (40), (41) and the fact that $f\left(r_{+}\right)=0$, one can obtain a Smarr-type formula as

$$
M(S, \mathbf{J}, Q)=\frac{\left[\left(n-1-\alpha^{2}\right) Z+\alpha^{2}-1\right] \mathbf{J}}{l\left(n-1-\alpha^{2}\right) \sqrt{Z(Z-1)}}
$$

where $\mathbf{J}=\sqrt{\sum_{i}^{k} J_{i}^{2}}$, and $Z=\Xi^{2}$ is the positive real root of the following equation:

$$
\begin{aligned}
\mathbf{J}+ & \frac{\beta^{2} l^{4-n}\left(\alpha^{2}+1\right)}{2 \pi(n-2)\left(n-1-\alpha^{2}\right)} \\
& \cdot b^{\alpha^{2}} \sqrt{Z(Z-1)}\left(\frac{4 S l^{n-3}}{\sqrt{Z}}\right)^{\left(n-1-\alpha^{2}\right) /(n-2)} \\
& \cdot\left\{\left(n-1-\alpha^{2}\right)\right. \\
& \cdot\left[\ln (-1+\sqrt{1+\zeta})-\ln \left(\frac{\zeta}{2}\right)+\sqrt{1+\zeta}\right] \\
& +(n-2) \\
& \cdot{ }_{2} F_{1}\left(\left[-\frac{1}{2}, \frac{\alpha^{2}-n+1}{2 n-4}\right],\left[\frac{\alpha^{2}+n-3}{2 n-4}\right],-\zeta\right) \\
& \left.+\frac{(n-1)(n-2)}{8 l^{2} \beta^{2}}\left(\alpha^{2}-n+1\right)+\alpha^{2}-2 n+3\right\} \\
& =0,
\end{aligned}
$$

where $\zeta=\pi^{2} Q^{2} /\left(S^{2} \beta^{2}\right)$. We can regard the parameters $S$, $\mathbf{J}$, and $Q$ as a complete set of extensive parameters for the mass $M(S, \mathbf{J}, Q)$ and define the intensive parameters conjugate to $S$, $J$, and $Q$. These parameters are, respectively, the temperature, the angular velocities, and the electric potential, which are defined as

$$
\begin{aligned}
T & =\left(\frac{\partial M}{\partial S}\right)_{J, Q}, \\
\Omega_{i} & =\left(\frac{\partial M}{\partial J_{i}}\right)_{S, Q}, \\
U & =\left(\frac{\partial M}{\partial Q}\right)_{S, \mathbf{J}} .
\end{aligned}
$$

Numerical calculations show that the intensive quantities calculated by (47) coincide with (36) and (45). Thus, these thermodynamics quantities satisfy the first law of thermodynamics

$$
d M=T d S+\sum_{i=1}^{k} \Omega_{i} d J_{i}+U d Q
$$

\section{Thermal Stability of the Black Branes in Canonical and Grand-Canonical Ensembles}

In this section, we intend to investigate thermal stability of our nonlinearly charged rotating black brane solutions in both canonical and grand-canonical ensembles. We know that the entropy of a thermally stable system is at local maximum. The aim of thermal stability analysis is to find the situations under which the system is stable thermally; that is, its entropy is a local maximum. Therefore, the 
stability of charged rotating black brane is studied in terms of entropy $S(M, Q, J)$. However, the thermal stability can also be discussed in terms of internal energy. When the entropy is at local maximum, the internal energy is at local minimum. Hence, we can equivalently analyse thermal stability in terms of Legendre transformation of entropy, namely, internal energy $M(S, Q, J)$. This analysis is commonly done by studying the determinant of the Hessian matrix of $M(S, Q, J)$ with respect to its extensive variables $X_{i}, \mathbf{H}_{X_{i} X_{j}}^{M}=\left[\partial^{2} M / \partial X_{i} \partial X_{j}\right]$ [84-86]. The positivity of $\mathbf{H}_{X_{i} X_{j}}^{M}$ shows that the system is thermally stable. The number of thermodynamic variables depends on the ensemble in which the system is studied. For instance, in canonical ensemble where the charge and angular momenta are fixed, the entropy is the only variable and consequently $\mathbf{H}_{X_{i} X_{j}}^{M}$ reduces to $\left(\partial^{2} M / \partial S^{2}\right)_{\mathrm{Q}, \mathrm{J}}$. Thus, in this ensemble, the positivity of $\left(\partial^{2} M / \partial S^{2}\right)_{\mathrm{Q}, \mathrm{J}}$ is sufficient to ensure the thermal stability of course in the ranges the temperature $T$ is positive as well. In grand-canonical ensemble $Q$ and $\mathbf{J}$ are no longer fixed.

Since the presence of charge does not change stable solutions to unstable ones [76, 77], we first study thermal stability for uncharged case; that is, $q \rightarrow 0$. In this case

$$
\begin{aligned}
& \left(\frac{\partial^{2} M}{\partial S^{2}}\right)_{\mathbf{J}} \\
& =\frac{(n-1)\left(\alpha^{2}+1\right)\left[\left(\Xi^{2}-1\right)\left(n-2 \alpha^{2}\right)+\Xi^{2}\left(1-\alpha^{2}\right)\right]}{\pi \Xi^{2} l^{5-n} b^{(n-4) \gamma / 2}\left[\left(\alpha^{2}+n-3\right) \Xi^{2}+1-\alpha^{2}\right]} \\
& \quad \cdot r_{+}^{\left(3-n-\alpha^{2}\right) /\left(\alpha^{2}+1\right)}, \\
& \mathbf{H}_{S \mathbf{J}}^{M}=\frac{16\left(1-\alpha^{2}\right) l^{2(n-4)} r_{+}^{2(2-n) /\left(\alpha^{2}+1\right)}}{b^{(n-2) \gamma} \Xi^{4}\left[\left(\Xi^{2}-1\right) \alpha^{2}+1+(n-3) \Xi^{2}\right]} .
\end{aligned}
$$

Since $\Xi^{2} \geq 1$, both (49) and $\mathbf{H}_{S \mathrm{~J}}^{M}$ are positive for $\alpha \leq$ 1 ; therefore the uncharged rotating solutions are stable in both canonical and grand-canonical ensembles provided $\alpha \leq$ 1. For this case, the temperature is also always positive as one can see from (37). As pointed out before, the charge cannot change thermal stability and therefore we always have thermally stable rotating black brane solutions for $\alpha \leq 1$. This fact is illustrated in Figures 3 and 4 for different values of $r_{+}$. The positivity of temperature for them is shown in Figure 5. For different $q$ 's, Figures 6 and 7 show that charge does not affect the thermal stability and therefore charged solutions are still stable for $\alpha \leq 1$. The positivity of $T$ for mentioned parameters in Figures 6 and 7 is shown in Figure 8.

Now, we discuss the stability for nonlinearly charged rotating black brane solutions for $\alpha>1$. One can see from (50) that $\alpha=1$ is the root of $\mathbf{H}_{S \mathrm{~J}}^{M}$. Numerical investigations show that $\alpha=1$ is the root of determinant of Hessian matrix in charged case too. Also, for $\alpha>1, \mathbf{H}_{S, Q, J}^{M}$ is always negative as $\mathbf{H}_{S \mathrm{~J}}^{M}$ obviously is (see (50)). Therefore, we have unstable solutions for $\alpha>1$ in grand-canonical ensemble. Figures 4 and 7 illustrate this fact. However, in canonical ensemble we have both stable and unstable solutions for $\alpha>1$. Figures 3 and 6 show that there is an $\alpha_{\max }(>1)$ that we have stable

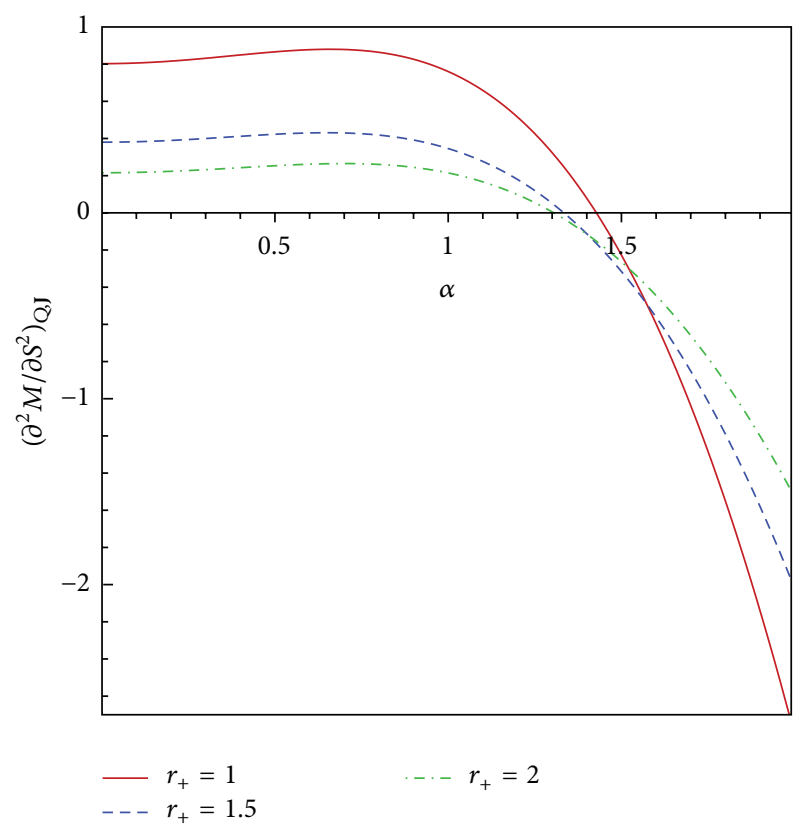

FIGURE 3: The behavior of $\left(\partial^{2} M / \partial S^{2}\right)_{\mathrm{Q}, \mathrm{J}}$ versus $\alpha$ with $l=b=1$, $q=0.8, \Xi=1.25, n=5$, and $\beta=2$.

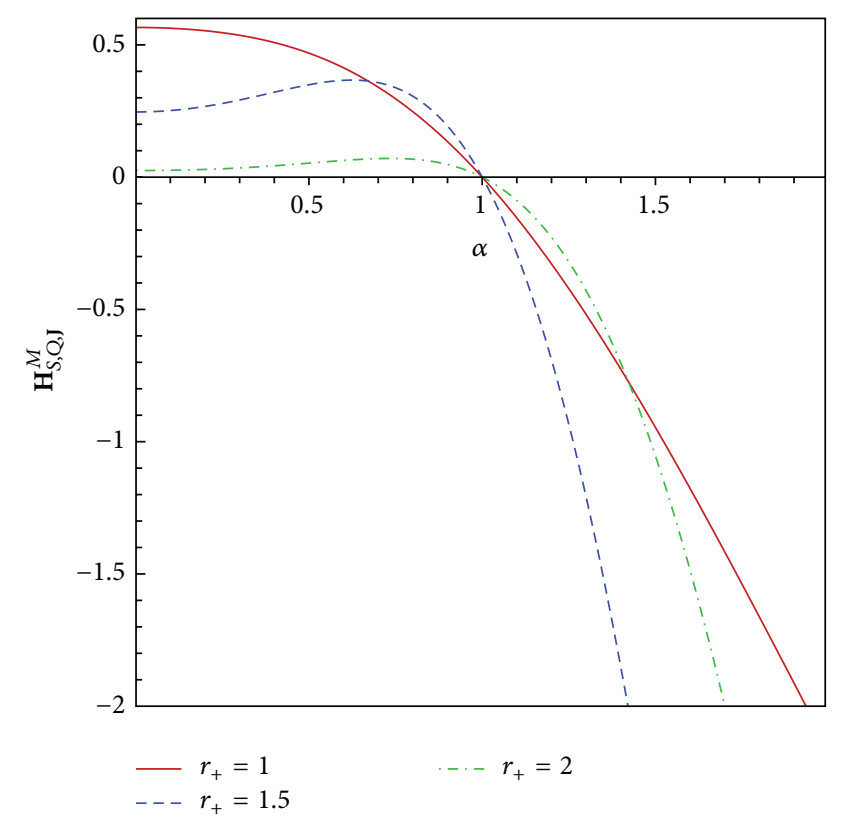

FIGURE 4: The behavior of $\mathbf{H}_{S, Q, J}^{M}$ versus $\alpha$ with $l=b=1, q=0.8$, $\Xi=1.25, n=5$, and $\beta=2$. Note that the curve corresponding to $r_{+}=1$ is rescaled by a factor $10^{-1}$.

solutions for values lower than it (note that $\alpha<\sqrt{n-1}$; see sentences above (38)). There is also $r_{+\max }$ that for $r_{+}>$ $r_{+\max }$ solutions are unstable (see Figure 9). The behavior of $\left(\partial^{2} M / \partial S^{2}\right)_{\mathrm{Q}, \mathrm{J}}$ in terms of $q$ and $\beta$ is depicted in Figures 10 and 11, respectively. These figures show that there are $q_{\min }$ and $\beta_{\min }$ that for values greater than them black branes are thermally stable. 


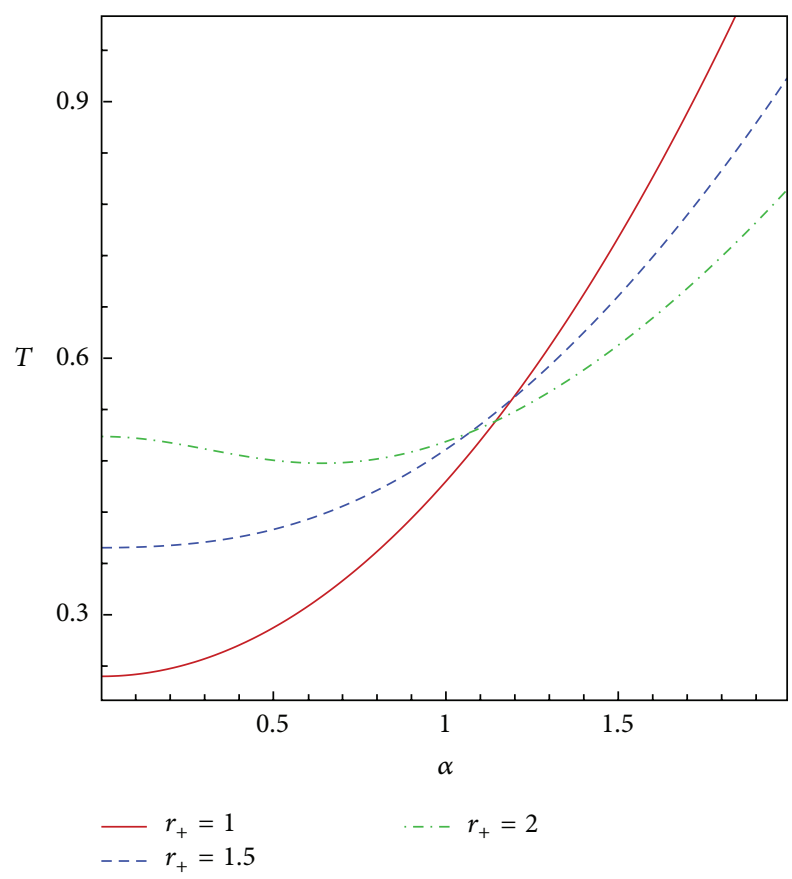

FIGURE 5: The behavior of $T$ versus $\alpha$ with $l=b=1, q=0.8$, $\Xi=1.25, n=5$, and $\beta=2$.

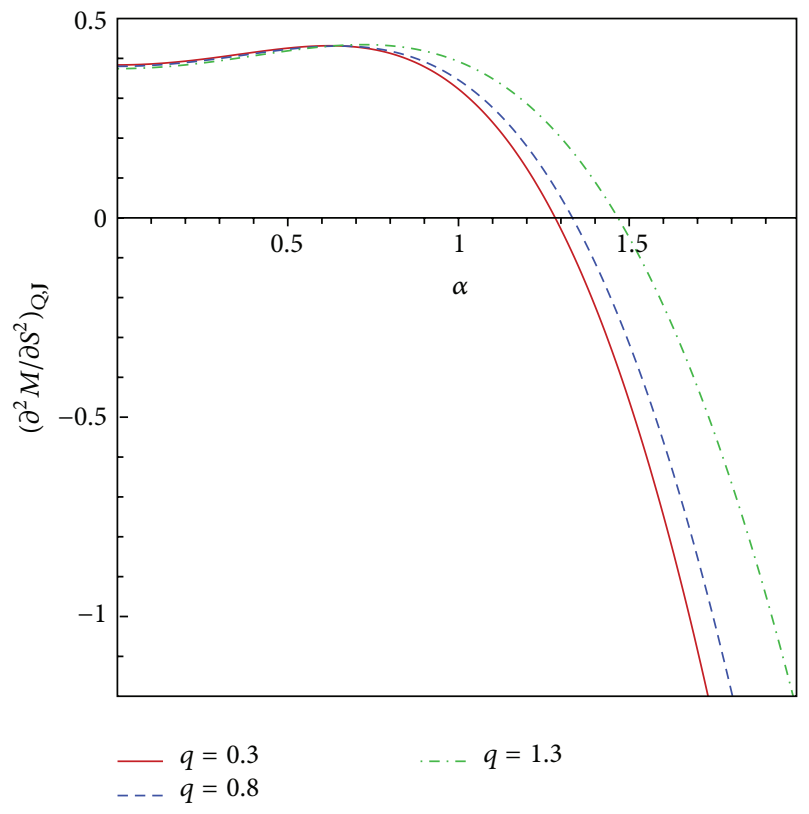

Figure 6: The behavior of $\left(\partial^{2} M / \partial S^{2}\right)_{\mathrm{Q}, \mathrm{J}}$ versus $\alpha$ with $l=b=1$, $r_{+}=1.5, \Xi=1.25, n=5$, and $\beta=2$.

\section{Conclusions and Discussions}

In this paper, we studied the higher dimensional action in the context of dilaton gravity and in the presence of the logarithmic nonlinear electrodynamics. By varying the action, we found the field equations of this theory. Then, we constructed a new class of charged, rotating black brane solutions, with $k=[(n-1) / 2]$ rotation parameters, in

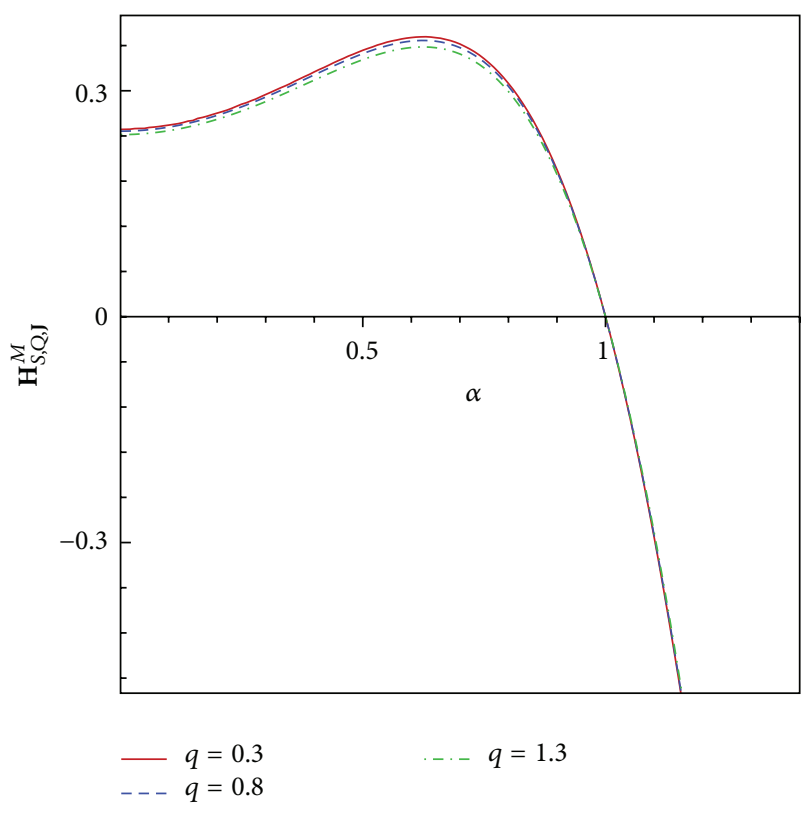

FIGURE 7: The behavior of $\mathbf{H}_{S, Q, J}^{M}$ versus $\alpha$ with $l=b=1, r_{+}=1.5$, $\Xi=1.25, n=5$, and $\beta=2$.

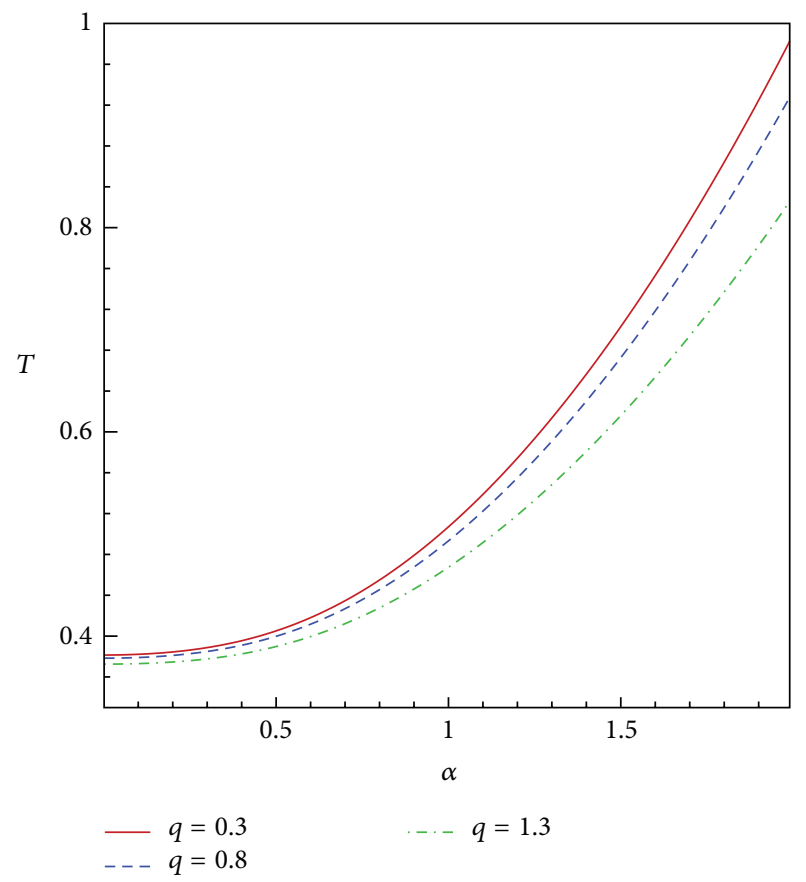

Figure 8: The behavior of $T$ versus $\alpha$ with $l=b=1, r_{+}=1.5$, $\Xi=1.25, n=5$, and $\beta=2$.

an arbitrary dimension. We found that the presence of the dilaton field changes the asymptotic behavior of the obtained solutions to be neither flat nor (A)dS. We presented the suitable counterterm which removes the divergences of the action in the presence of the dilaton field. In the absence of a nontrivial dilaton $(\alpha=\gamma=0)$, these solutions reduce to the asymptotically AdS charged rotating black brane 


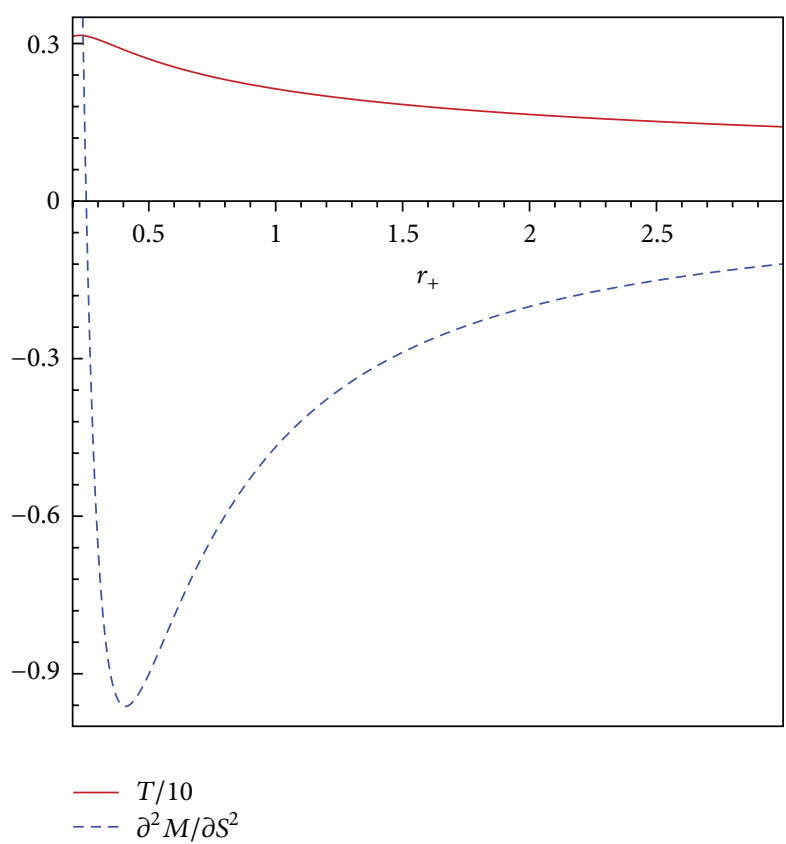

FIGURE 9: The behaviors of $10^{-1} T$ and $\left(\partial^{2} M / \partial S^{2}\right)_{\mathrm{QJ}}$ versus $r_{+}$with $l=1, b=2, \alpha=1.5, \Xi=1.25, n=5, \beta=5$, and $q=1.1$.

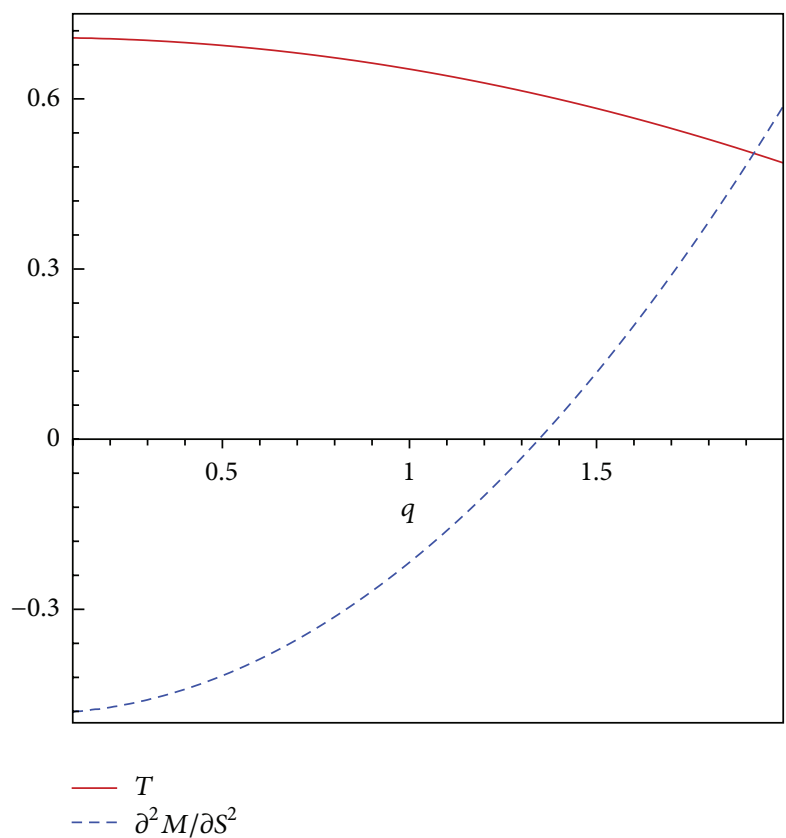

Figure 10: The behaviors of $T$ and $\left(\partial^{2} M / \partial S^{2}\right)_{\mathrm{Q}, \mathrm{J}}$ versus $q$ with $l=$ $b=1, \alpha=1.5, \Xi=1.25, n=5, \beta=5$, and $r_{+}=1.5$.

solutions of Einstein theory in the presence of logarithmic nonlinear electrodynamics $[52,53]$. When $\beta \rightarrow \infty$, these solutions reduce to the charged rotating dilaton black brane solutions given in [71]. We also calculated the conserved and thermodynamic quantities of the spacetime such as mass, angular momentum, temperature, entropy, and electric potential and checked that the first law of thermodynamics holds on the black brane horizon.

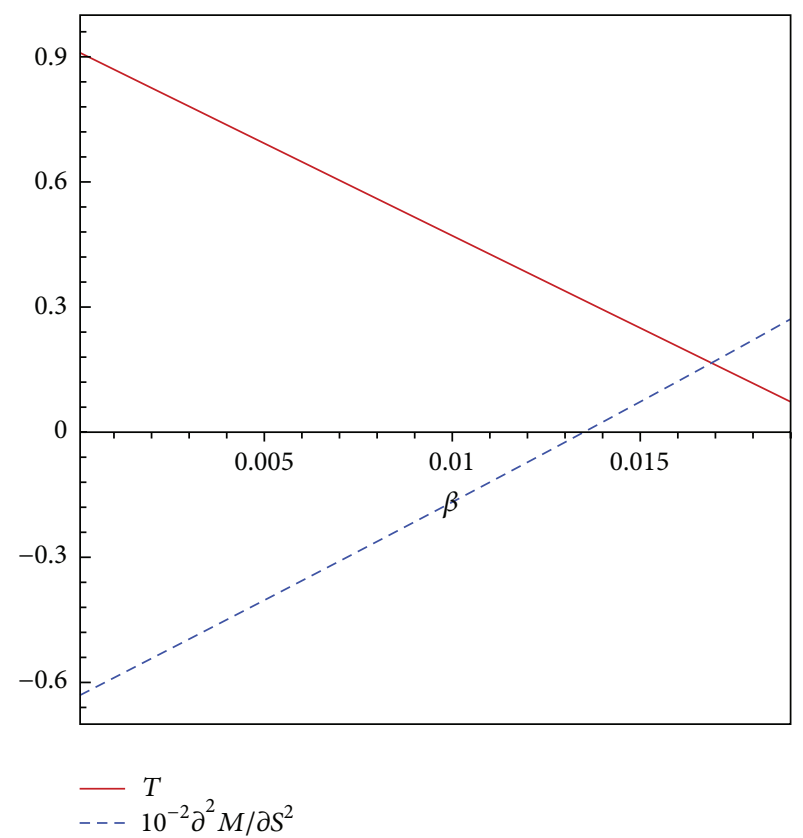

FIGURE 11: The behaviors of $T$ and $10^{-2}\left(\partial^{2} M / \partial S^{2}\right)_{\text {Q,J }}$ versus $\beta$ with $l=1, b=0.5, \alpha=3, \Xi=1.25, n=6, q=10$, and $r_{+}=1$.

Then, we explored thermal stability of the nonlinearly charged rotating black brane solutions in both canonical and grand-canonical ensembles. We found that in both ensembles the solutions are thermally stable for $\alpha \leq 1$, while for $\alpha>$ 1 the solutions are always thermally unstable in the grandcanonical ensemble, where $\alpha$ is the dilaton-electromagnetic coupling constant. In the canonical ensemble, however, we can have both stable and unstable solutions for $\alpha>1$. We found that, in this ensemble, there is $\alpha_{\max }>1$ for which the solutions are thermally stable provided $\alpha<\alpha_{\max }$. The pointed out results imply that the thermal stability is ensembledependent and $\alpha$ influences the stability under thermal perturbations. These results are expectable since different ensembles allow different sets of quantities to be variable and a thermally stable system is one which is stable under varying variable quantities. On the other hand, values of conserved and thermodynamic quantities depend on values of parameters such as $\alpha$ and therefore the fact that dilatonelectromagnetic coupling has direct effect on thermal stability of the system seems natural.

It is notable to mention that, in this paper, we only constructed the charged rotating dilaton black branes of nonlinear electrodynamics with flat horizon. One can try to construct the rotating dilaton black holes of this theory with curved horizon. One can also investigate the thermodynamic geometry of these solutions. The latter issue is now under investigation and the results will be presented elsewhere.

\section{Competing Interests}

The authors declare no competing interests for the present paper. 


\section{Acknowledgments}

The authors thank the Research Council of Shiraz University. This work has been supported financially by Research Institute for Astronomy \& Astrophysics of Maragha (RIAAM), Iran.

\section{References}

[1] J. D. Bekenstein, "Black holes and entropy," Physical Review D, vol. 7, pp. 2333-2346, 1973.

[2] S. W. Hawking, "Black hole explosions?", Nature, vol. 248, no. 5443, pp. 30-31, 1974.

[3] A. G. Riess, A. V. Filippenko, P. Challis et al., "Observational evidence from supernovae for an accelerating universe and a cosmological constant," The Astronomical Journal, vol. 116, no. 3, pp. 1009-1038, 1998.

[4] S. Perlmutter, G. Aldering, G. Goldhaber et al., "Measurements of $\Omega$ and $\Lambda$ from 42 high-redshift supernovae," The Astrophysical Journal, vol. 517, no. 2, p. 565, 1999.

[5] J. L. Tonry, B. P. Schmidt, B. Barris et al., "Cosmological results from high- $z$ supernovae," The Astrophysical Journal, vol. 594, no. 1, article 1, 2003.

[6] A. T. Lee, P. Ade, A. Balbi et al., "A high spatial resolution analysis of the MAXIMA-1 cosmic microwave background anisotropy data," The Astrophysical Journal Letters, vol. 561, no. 1, p. L1, 2001.

[7] C. B. Nettereld, P. A. R. Ade, J. J. Bock et al., "A measurement by BOOMERANG of multiple peaks in the angular power spectrum of the cosmic microwave background," The Astrophysical Journal, vol. 571, no. 2, pp. 604-614, 2002.

[8] N. W. Halverson, E. M. Leitch, C. Pryke et al., "Degree angular scale interferometer first results: a measurement of the cosmic microwave background angular power spectrum," The Astrophysical Journal, vol. 568, no. 1, pp. 38-45, 2002.

[9] D. N. Spergel, L. Verde, H. V. Peiris et al., "First-year Wilkinson Microwave Anisotropy Probe (WMAP) observations: determination of cosmological parameters," The Astrophysical Journal Supplement Series, vol. 148, no. 1, article 175, 2003.

[10] M. B. Green, J. H. Schwarz, and E. Witten, Superstring Theory, Cambridge University Press, Cambridge, UK, 1987.

[11] G. W. Gibbons and K.-i. Maeda, "Black holes and membranes in higher-dimensional theories with dilaton fields," Nuclear Physics B, vol. 298, no. 4, pp. 741-775, 1988.

[12] T. Koikawa and M. Yoshimura, "Dilaton fields and event horizon," Physics Letters B, vol. 189, no. 1-2, pp. 29-33, 1987.

[13] D. Brill and G. T. Horowitz, "Negative energy in string theory," Physics Letters B, vol. 262, no. 4, pp. 437-443, 1991.

[14] D. Garfinkle, G. T. Horowitz, and A. Strominger, "Charged black holes in string theory," Physical Review D, vol. 43, no. 10, pp. 3140-3143, 1991.

[15] R. Gregory and J. A. Harvey, "Black holes with a massive dilaton," Physical Review D, vol. 47, no. 6, pp. 2411-2422, 1993.

[16] M. Rakhmanov, "Dilaton black holes with electric charge," Physical Review D, vol. 50, no. 8, pp. 5155-5163, 1994.

[17] S. Mignemi and D. L. Wiltshire, "Spherically symmetric solutions in dimensionally reduced spacetimes," Classical and Quantum Gravity, vol. 6, no. 7, pp. 987-1002, 1989.
[18] D. L. Wiltshire, "Spherically symmetric solutions in dimensionally reduced spacetimes with a higher-dimensional cosmological constant," Physical Review D, vol. 44, no. 4, pp. 1100-1114, 1991.

[19] S. Mignemi and D. L. Wiltshire, "Black holes in higherderivative gravity theories," Physical Review D, vol. 46, no. 4, pp. 1475-1506, 1992.

[20] S. J. Poletti and D. L. Wiltshire, "Global properties of static spherically symmetric charged dilaton spacetimes with a Liouville potential," Physical Review. D. Third Series, vol. 50, no. 12, pp. 7260-7270, 1994.

[21] R.-G. Cai, J.-Y. Ji, and K.-S. Soh, “Topological dilaton black holes," Physical Review D, vol. 57, no. 10, pp. 6547-6550, 1998.

[22] R.-G. Cai and Y.-Z. Zhang, "Holography and brane cosmology in domain wall backgrounds," Physical Review D, vol. 64, no. 10, Article ID 104015, 8 pages, 2001.

[23] S. S. Yazadjiev, "Non-asymptotically flat, non-dS/AdS Dyonic black holes in dilaton gravity," Classical and Quantum Gravity, vol. 22, no. 19, pp. 3875-3889, 2005.

[24] K. C. Chan, J. H. Horne, and R. B. Mann, "Charged dilaton black holes with unusual asymptotics," Nuclear Physics B, vol. 447, no. 2-3, pp. 441-461, 1995.

[25] G. Clément, D. Gal'tsov, and C. Leygnac, "Linear dilaton black holes," Physical Review D, vol. 67, no. 2, Article ID 024012, 14 pages, 2003.

[26] G. Clément and C. Leygnac, "Non-asymptotically flat, non-AdS dilaton black holes," Physical Review D, vol. 70, no. 8, Article ID 084018, 17 pages, 2004.

[27] A. Sheykhi, "Thermodynamics of charged topological dilaton black holes," Physical Review D, vol. 76, no. 12, Article ID 124025, 8 pages, 2007.

[28] A. Sheykhi and N. Riazi, "Rotating solutions of EinsteinMaxwell-dilaton gravity with unusual asymptotics," International Journal of Theoretical Physics, vol. 45, no. 12, pp. 24532463, 2006.

[29] A. Sheykhi, M. H. Dehghani, N. Riazi, and J. Pakravan, "Thermodynamics of rotating solutions in $(n+1)$-dimensional Einstein-Maxwell-dilaton gravity," Physical Review D, vol. 74, no. 8, Article ID 084016, 8 pages, 2006.

[30] A. Sheykhi, "Rotating black holes in Einstein-Maxwell-dilaton gravity," Physical Review D, vol. 77, no. 10, Article ID 104022, 7 pages, 2008.

[31] M. Kord Zangeneh, A. Sheykhi, and M. H. Dehghani, “Thermodynamics of higher dimensional topological dilation black holes with a power-law Maxwell field," Physical Review D, vol. 91, no. 4, Article ID 044035, 11 pages, 2015.

[32] A. Sheykhi, "Thermodynamical properties of topological borninfeld-dilaton black holes," International Journal of Modern Physics D, vol. 18, no. 1, pp. 25-42, 2009.

[33] S. H. Hendi, "Asymptotic charged BTZ black hole solutions," Journal of High Energy Physics, vol. 2012, no. 3, article 65, 2012.

[34] A. Sheykhi and A. Kazemi, "Higher dimensional dilaton black holes in the presence of exponential nonlinear electrodynamics," Physical Review D, vol. 90, no. 4, Article ID 044028, 2014.

[35] M. Kord Zangeneh, A. Sheykhi, and M. H. Dehghani, "Thermodynamics of charged rotating dilaton black branes with powerlaw Maxwell field," European Physical Journal C, vol. 75, article 497, 2015.

[36] M. H. Dehghani, S. H. Hendi, A. Sheykhi, and H. Rastegar Sedehi, "Thermodynamics of rotating black branes in $(n+1)$ dimensional Einstein-Born-Infeld-dilaton gravity," Journal of 
Cosmology and Astroparticle Physics, vol. 2007, no. 2, article 020, 2007.

[37] A. Sheykhi and S. H. Hendi, "Thermodynamics of rotating black branes in higher dimensional Einstein-nonlinear electrodynamics-dilaton gravity," Canadian Journal of Physics, vol. 94, no. 1, pp. 58-70, 2016.

[38] M. Born and L. Infeld, "Foundations of the new field theory," Proceedings of the Royal Society of London A, vol. 144, pp. 425451, 1934.

[39] E. S. Fradkin and A. A. Tseytlin, "Nonlinear electrodynamics from quantized strings," Physics Letters B, vol. 163, no. 1-4, pp. 123-130, 1985.

[40] E. Bergshoeff, E. Sezgin, C. N. Pope, and P. K. Townsend, "The Born-Infeld action from conformal invariance of the open superstring," Physics Letters B, vol. 188, no. 1, pp. 70-74, 1987.

[41] C. G. Callan, C. Lovelace, C. R. Nappi, and S. A. Yost, "Loop corrections to superstring equations of motion," Nuclear Physics $B$, vol. 308, no. 2-3, pp. 221-284, 1988.

[42] O. D. Andreev and A. A. Tseytlin, "Partition-function representation for the open superstring effective action: cancellation of Möbius infinites and derivative corrections to Born-Infeld lagrangian," Nuclear Physics B, vol. 311, no. 1, pp. 205-252, 1988.

[43] R. Leigh, "Dirac-born-infeld action from dirichlet $\sigma$-model," Modern Physics Letters A, vol. 4, pp. 2767-2772, 1989.

[44] H. H. Soleng, "Charged black points in general relativity coupled to the logarithmic U(1) gauge theory," Physical Review D, vol. 52, no. 10, pp. 6178-6181, 1995.

[45] R. G. Cai and Y. W. Sun, "Shear viscosity from AdS Born-Infeld black holes," Journal of High Energy Physics, vol. 9, p. 115, 2008.

[46] J. Jing and S. Chen, "Holographic superconductors in the BornInfeld electrodynamics," Physics Letters B, vol. 686, no. 1, pp. 6871, 2010.

[47] R. Gregory, S. Kanno, and J. Soda, "Holographic superconductors with higher curvature corrections," Journal of High Energy Physics, vol. 2009, no. 10, p. 10, 2009.

[48] R. R. Metsaev, M. A. Rahmanov, and A. A. Tseytlin, "The borninfeld action as the effective action in the open superstring theory," Physics Letters B, vol. 193, no. 2-3, pp. 207-212, 1987.

[49] A. A. Tseytlin, "On non-abelian generalisation of the BornInfeld action in string theory," Nuclear Physics B, vol. 501, no. 1, pp. 41-52, 1997.

[50] D. Brecher and M. J. Perry, "Bound states of D-branes and the non-abelian Born-Infeld action," Nuclear Physics B, vol. 527, no. 1-2, pp. 121-141, 1998.

[51] R. G. Leigh, "Dirac-born-infeld action from dirichlet $\sigma$-model," Modern Physics Letters A, vol. 4, no. 28, p. 2767, 1989.

[52] S. H. Hendi, "Asymptotic Reissner-Nordström black holes," Annals of Physics, vol. 333, pp. 282-289, 2013.

[53] S. H. Hendi and A. Sheykhi, "Charged rotating black string in gravitating nonlinear electromagnetic fields," Physical Review $D$, vol. 88, no. 4, Article ID 044044, 2013.

[54] S. H. Hendi and M. Sepehri Rad, "Five dimensional MyersPerry black holes with nonlinear electrodynamics," Physical Review D, vol. 90, no. 8, Article ID 084051, 2014.

[55] S. H. Hendi and M. Allahverdizadeh, "Slowly rotating black holes with nonlinear electrodynamics," Advances in High Energy Physics, vol. 2014, Article ID 390101, 7 pages, 2014.

[56] A. Sheykhi and S. Hajkhalili, "Dilaton black holes coupled to nonlinear electrodynamic field," Physical Review D, vol. 89, no. 10, Article ID 104019, 2014.
[57] A. Sheykhi and A. Kazemi, "Higher dimensional dilaton black holes in the presence of exponential nonlinear electrodynamics," Physical Review D, vol. 90, no. 4, Article ID 044028, 2014.

[58] A. Sheykhi and N. Riazi, "Thermodynamics of black holes in $(n+1)$-dimensional Einstein-Born-Infeld-dilaton gravity," Physical Review D, vol. 75, Article ID 024021, 2007.

[59] M. H. Dehghani, A. Sheykhi, and S. H. Hendi, "Magnetic strings in Einstein-Born-Infeld-dilaton gravity," Physics Letters B, vol. 659, no. 3, pp. 476-482, 2008.

[60] S. W. Hawking and S. F. Ross, "Duality between electric and magnetic black holes," Physical Review D, vol. 52, no. 10, pp. 5865-5876, 1995.

[61] M. H. Dehghani, C. Shakuri, and M. H. Vahidinia, "Lifshitz black brane thermodynamics in the presence of a nonlinear electromagnetic field," Physical Review D, vol. 87, no. 8, Article ID 084013, 2013.

[62] M. H. Dehghani, A. Sheykhi, and S. E. Sadati, "Thermodynamics of nonlinear charged Lifshitz black branes with hyperscaling violation," Physical Review D, vol. 91, no. 12, Article ID 124073, 8 pages, 2015.

[63] J. D. Brown and J. W. York, "Quasilocal energy and conserved charges derived from the gravitational action," Physical Review $D$, vol. 47, no. 4, pp. 1407-1419, 1993.

[64] J. Maldacena, "The large $N$ limit of superconformal field theories and supergravity," Advances in Theoretical and Mathematical Physics, vol. 2, no. 2, pp. 231-252, 1998.

[65] E. Witten, "Anti de Sitter space and holography," Advances in Theoretical and Mathematical Physics, vol. 2, no. 2, pp. 253-291, 1998.

[66] O. Aharony, S. S. Gubser, J. Maldacena, H. Ooguri, and Y. $\mathrm{Oz}$, "Large $N$ field theories, string theory and gravity," Physics Reports. A Review Section of Physics Letters, vol. 323, no. 3-4, pp. 183-386, 2000.

[67] V. Balasubramanian and P. Kraus, "A stress tensor for anti-de Sitter gravity," Communications in Mathematical Physics, vol. 208, no. 2, pp. 413-428, 1999.

[68] H. J. Boonstra, K. Skenderis, and P. K. Townsend, "The domainwall/QFT correspondence," Journal of High Energy Physics, vol. 1999, no. 1, p. 3, 1999.

[69] K. Behrndt, E. Bergshoeff, R. Halbersma, and J. P. Van der Schaar, "On domain-wall/QFT dualities in various dimensions," Classical and Quantum Gravity, vol. 16, no. 11, pp. 3517-3552, 1999.

[70] R.-G. Cai and N. Ohta, "Surface counterterms and boundary stress-energy tensors for asymptotically non-anti-de Sitter spaces," Physical Review D, vol. 62, no. 2, Article ID 024006, 12 pages, 2000.

[71] A. Sheykhi, M. H. Dehghani, N. Riazi, and J. Pakravan, "Thermodynamics of rotating solutions in $(n+1)$-dimensional Einstein-Maxwell-dilaton gravity," Physical Review D, vol. 74, no. 8, Article ID 084016, 2006.

[72] A. M. Awad, "Higher-dimensional charged rotating solutions in (A)dS spacetimes," Classical and Quantum Gravity, vol. 20, no. 13, pp. 2827-2833, 2003.

[73] J. P. S. Lemos and V. T. Zanchin, "Rotating charged black strings and three-dimensional black holes," Physical Review D, vol. 54, no. 6, pp. 3840-3853, 1996.

[74] M. Abramowitz and I. A. Stegun, Handbook of Mathematical Functions, Dover, New York, NY, USA, 1972.

[75] R. M. Corless, G. H. Gonnet, D. E. Hare, D. J. Jeffrey, and D. E. Knuth, "On the LambertW function," Advances in Computational Mathematics, vol. 5, no. 1, pp. 329-359, 1996. 
[76] M. H. Dehghani, "Thermodynamics of rotating charged black strings and (A)dS/CFT correspondence," Physical Review D, vol. 66, no. 4, Article ID 044006, 6 pages, 2002.

[77] M. H. Dehghani and A. Khodam-Mohammadi, "Thermodynamics of a $d$-dimensional charged rotating black brane and the AdS/CFT correspondence," Physical Review D, vol. 67, no. 8, Article ID 084006, 7 pages, 2003.

[78] J. D. Beckenstein, "Black holes and entropy," Physical Review D, vol. 7, no. 8, Article ID 2333, 1973.

[79] G. W. Gibbons and S. W. Hawking, "Cosmological event horizons, thermodynamics, and particle creation," Physical Review D, vol. 15, no. 10, pp. 2738-2751, 1977.

[80] C. J. Hunter, "Action of instantons with a nut charge," Physical Review D, vol. 59, no. 2, Article ID 024009, 123 pages, 1999.

[81] S. W. Hawking, C. J. Hunter, and D. N. Page, "NUT charge, antide Sitter space, and entropy," Physical Review D, vol. 59, no. 4, Article ID 044033, 6 pages, 1999.

[82] R. B. Mann, "Misner string entropy," Physical Review D, vol. 60, no. 10, Article ID 104047, 5 pages, 1999.

[83] R. B. Mann, "Entropy of rotating Misner string spacetimes," Physical Review D, vol. 61, no. 8, Article ID 084013, 6 pages, 2000.

[84] M. Cvetic and S. S. Gubser, "Phases of R-charged black holes, spinning branes and strongly coupled gauge theories," Journal of High Energy Physics, vol. 1999, no. 4, p. 24, 1999.

[85] M. M. Caldarelli, G. Cognola, and D. Klemm, "Thermodynamics of Kerr-Newman-AdS black holes and conformal field theories," Classical and Quantum Gravity, vol. 17, no. 2, pp. 399420, 2000.

[86] S. S. Gubser and I. Mitra, "The evolution of unstable black holes in anti-de Sitter space," Journal of High Energy Physics, vol. 2001, no. 8 , p. $18,2001$. 

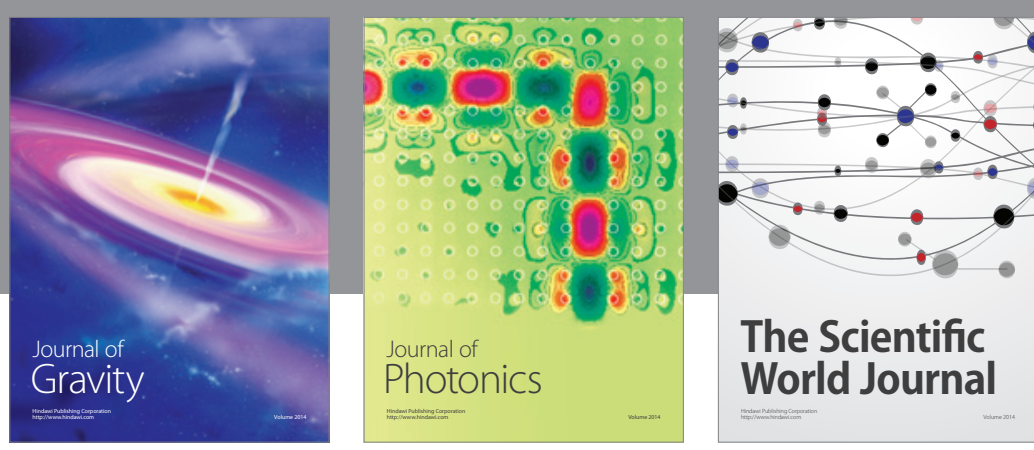

The Scientific World Journal
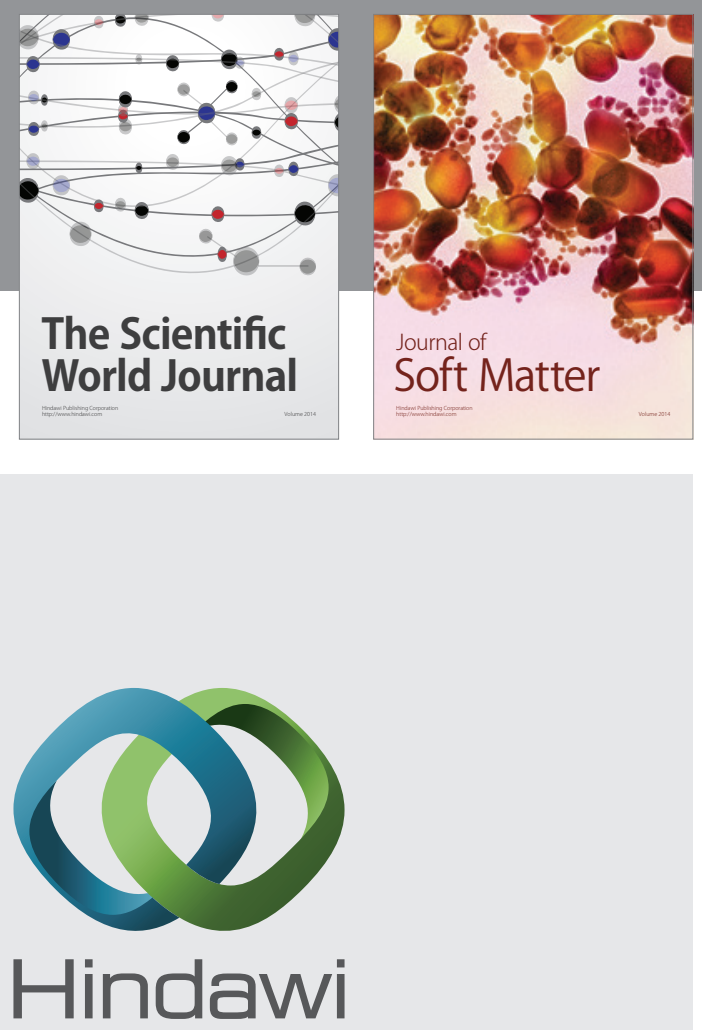

Submit your manuscripts at

http://www.hindawi.com

nternational Journal of

Statistical Mechanics
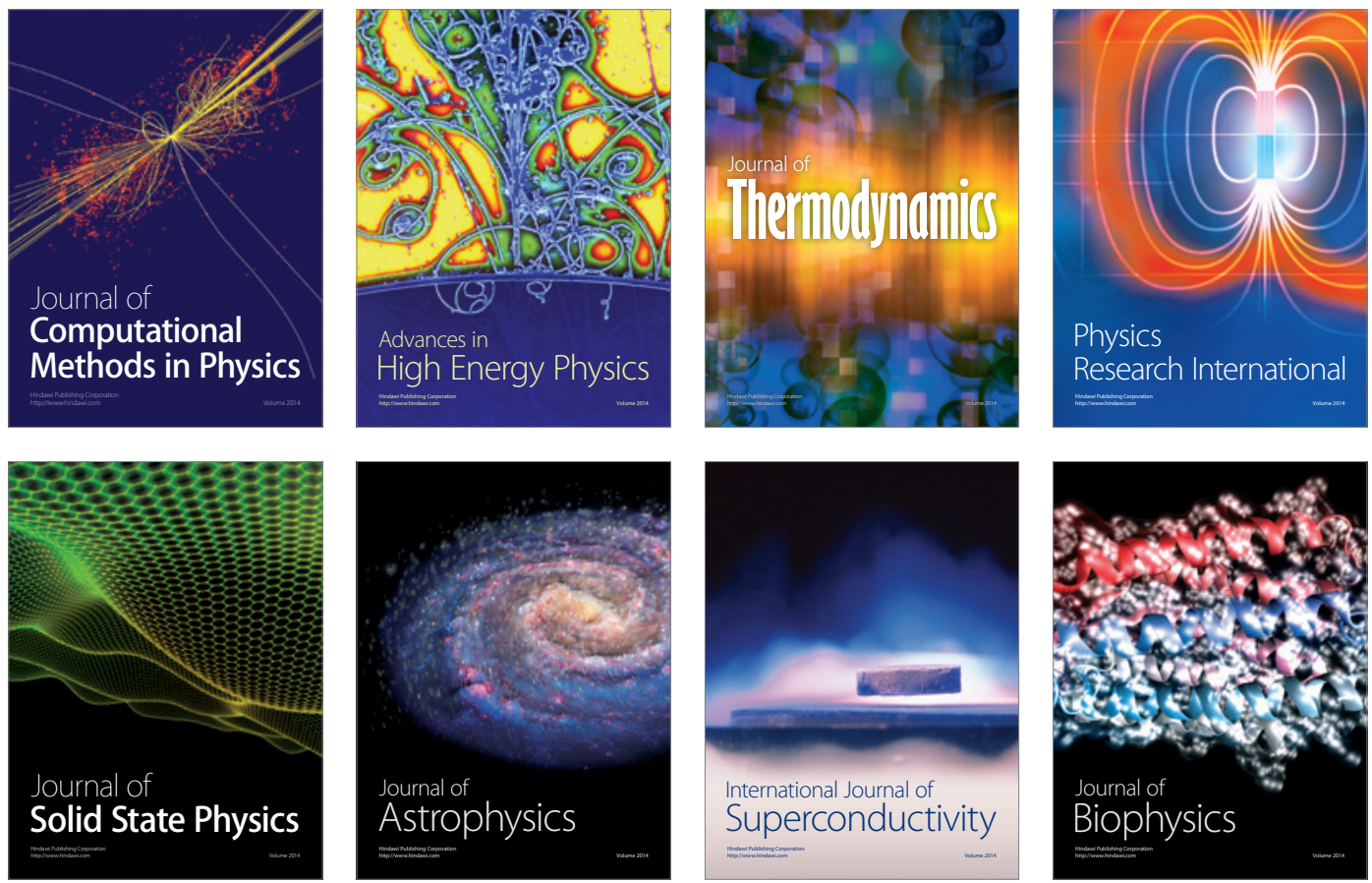
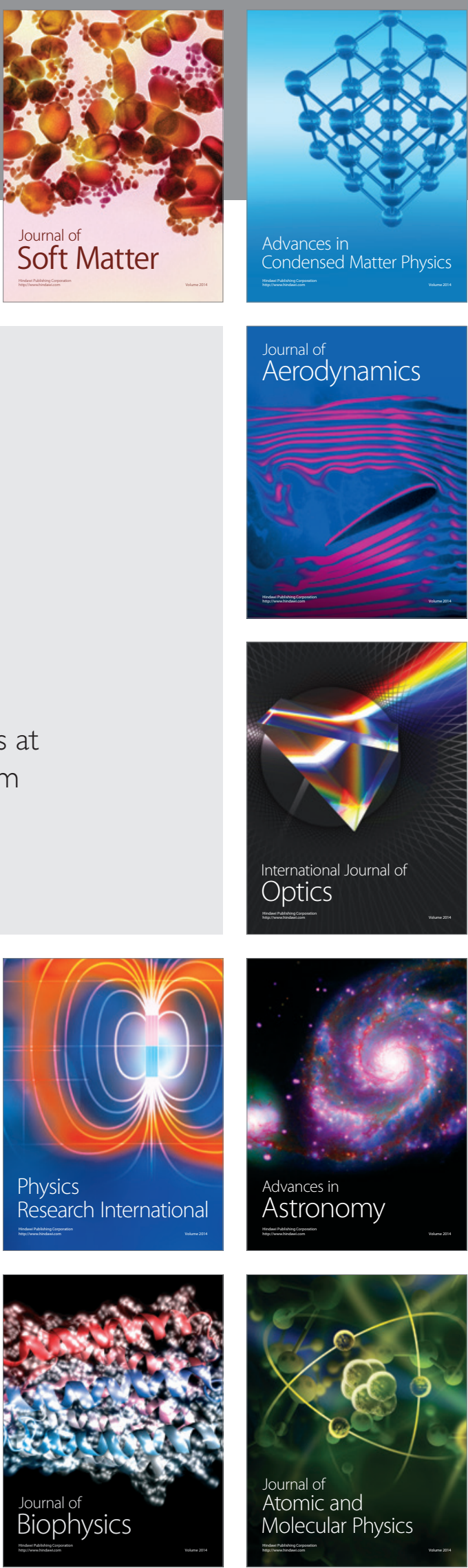